IJMMS 2003:2, 75-85

PII. S0161171203202295

http://ijmms.hindawi.com

(c) Hindawi Publishing Corp.

\title{
MATRIX MEASURE AND APPLICATION TO STABILITY OF MATRICES AND INTERVAL DYNAMICAL SYSTEMS
}

\author{
ZIAD ZAHREDDINE
}

Received 27 February 2002

\begin{abstract}
Using some properties of the matrix measure, we obtain a general condition for the stability of a convex hull of matrices that will be applied to study the stability of interval dynamical systems. Some classical results from stability theory are reproduced and extended. We present a relationship between the matrix measure and the real parts of the eigenvalues that make it possible to obtain stability criteria.
\end{abstract}

2000 Mathematics Subject Classification: 15A48, 37C75, 93D20, 93D09.

1. Introduction. Matrix stability plays an important role in mathematical analysis and its numerous applications to the areas of control theory, differential equations, and linear algebra. The concept of stability itself can be defined in many different ways depending on the context. Of special importance are stable (semistable) matrices - those whose eigenvalues have negative (nonpositive) real parts. A matrix $M$ is $D$-stable if $D \cdot M$ is stable for every choice of a positive diagonal matrix $D$. The notion of $D$-stability of a real square matrix was introduced mathematical economists Arrow and MacManus [2] in studying stability of equilibria in competitive market dynamic models. Relations between stability and $D$-stability were considered in [2, 7]. Another important stability concept is that of strong stability that arises when diffusion models of biological systems are linearized at a constant equilibrium. Solutions to most of the above problems depend on the stability of linear dynamical system of the form $d x / d t=A \cdot X$, which is studied on the basis of qualitative data on the entries of the matrix as in [13].

The stability of a linear dynamical system is determined by the eigenvalues of the system matrix. When the parameters in the system matrix are uncertain, we are interested in the stability of the matrix given this uncertainty. First studies in this area were realized by Chebyshev and Markov (see a survey of their works in [4]). The most interesting case is when the uncertainty in each parameter in the system matrix is modeled by an interval, that is, the lower and upper bounds are known. A natural conjecture would be that the family of system matrices is stable if and only if all the vertex matrices are stable. In $[8,9]$, sufficient conditions have been obtained, for which the above conjecture is true in the discrete case. In [5], an extension of Gershgorin's circle theorem 
was used to obtain a simple sufficient condition for the stability of the family of system matrices. The same theorem was also used in [1] to obtain more sufficient conditions. When the vertices of the matrix family are symmetric, the corresponding uncertain dynamical systems are stable if and only if the vertex matrices are stable, see [12]. In [10], Kharitonov presented a necessary and sufficient condition for the stability of dynamical systems whose parametric uncertainty is restricted to a rectangular domain. A more general version was discussed in [14], where the rectangular domain was replaced by a pathwise connected region $\Gamma$ in the complex plane. The concept of $\Gamma$-stability was then introduced, and necessary and sufficient conditions for the $\Gamma$-stability of a family of systems of differential equations were established. The results of [14] were generalized in [11].

In this paper, we prove a very general sufficient condition for a matrix family such that the stability of a finitely many well-chosen matrices guarantees stability of the convex hull and positive cone of the whole matrix family. This result implies the results in $[1,8,12]$. In some special cases dealing with the stability of interval dynamical systems, the condition becomes necessary as well as sufficient. Illustrative examples will be given.

2. Main results. Consider the convex hull of $n \times n$ matrices in the set $\mathbb{R}^{n \times n}$ described by

$$
C=\left\{M \mid M=\sum_{j=1}^{m} \gamma_{j} M_{j}, \gamma_{j} \geq 0, \sum_{j=1}^{m} \gamma_{j}=1\right\} .
$$

The matrix $M$ is said to be stable if all of its eigenvalues have negative real parts. We say that $C$ is stable if each matrix in $C$ is stable. In what follows, Re denotes the real part of a complex number and $\lambda(M)$ or $\lambda_{j}(M)$ denotes the eigenvalue of the matrix $M$. To state our results, some definitions are required.

Let $|x|$ denote a vector norm of $x$ on $\mathbb{C}^{n}$, and let $\|M\|$ be the induced matrix norm of $M$ given by the vector norm $|\cdot|$. Let $\mu(M)$ be the matrix measure of $M$ defined as

$$
\mu(M)=\lim _{\theta \rightarrow 0^{+}} \frac{\|I+\theta M\|-I}{\theta},
$$

where $I$ is the identity matrix. The following well-known Coppel inequality [3] gives an upper bound for the magnitude of the solution of a differential equation $\dot{y}(t)=M(t) y(t)$ in terms of the matrix measure

$$
\|y(t)\| \leq\left\|y\left(t_{0}\right)\right\| \exp \left(\int_{t_{0}}^{t} \mu(M(\tau)) d \tau\right),
$$

which proves useful in the discussions to follow. The next lemma may be found in [3]. 
LEMMA 2.1. The matrix measure $\mu(M)$ is well defined for any norm and has the following properties:

(a) $\mu$ is convex on $\mathbb{C}^{n \times n}$, that is, for any $\gamma_{j} \geq 0(1 \leq j \leq k), \sum_{j=1}^{k} \gamma_{j}=1$, and any matrices $M_{j}(1 \leq j \leq k)$, we have

$$
\mu\left(\sum_{j=1}^{k} \gamma_{j} M_{j}\right) \leq \sum_{j=1}^{k} \gamma_{j} \mu\left(M_{j}\right)
$$

(b) for any norm and any $M$, we have

$$
-\|M\| \leq-\mu(-M) \leq \operatorname{Re} \lambda(M) \leq \mu(M) \leq\|M\| ;
$$

(c) for the 1-norm $|x|_{1}=\sum_{j=1}^{n}\left|x_{j}\right|$, the induced matrix measure $\mu_{1}$ is given by

$$
\mu_{1}(M)=\max _{j}\left[\operatorname{Re}\left(m_{j j}\right)+\sum_{i \neq j}\left|m_{i j}\right|\right],
$$

for the 2-norm $|x|_{2}=\left(\sum_{j=1}^{n}\left|x_{j}\right|^{2}\right)^{1 / 2}$, the induced matrix measure $\mu_{2}$ is given by

$$
\mu_{2}(M)=\max _{i}\left[\frac{\lambda_{i}\left(M+M^{*}\right)}{2}\right]
$$

and for the $\infty$-norm $|x|_{\infty}=\max _{1 \leq j \leq n}\left|x_{j}\right|$, the induced matrix measure is given by

$$
\mu_{\infty}(M)=\max _{i}\left[\operatorname{Re}\left(m_{i i}\right)+\sum_{j \neq i}\left|m_{i j}\right|\right]
$$

(d) for any nonsingular matrix $N$ and any vector norm $\|\cdot\|$, with the induced matrix measure $\mu,\|N x\|$ defines another vector norm and its induced matrix measure $\mu_{N}$ is given by

$$
\mu_{N}(M)=\mu\left(N M N^{-1}\right)
$$

THEOREM 2.2. If there exists a norm such that $\mu\left(M_{j}\right)<0(1 \leq j \leq m)$, then $C$ is stable.

Proof. Let $M$ be any matrix in $C$, then there exist $\gamma_{j} \geq 0$ and $\sum_{j=1}^{m} \gamma_{j}=1$, so that $M=\sum_{j=1}^{m} \gamma_{j} M_{j}$. Since $\mu$ is convex and $M \in C$, we have

$$
\mu(M) \leq \sum_{j=1}^{m} \gamma_{j} \mu\left(M_{j}\right)<0 .
$$


Then by Lemma 2.1(b), we have $\operatorname{Re} \lambda_{j}(M) \leq \mu(M)<0$, which implies that $M$ is stable. Therefore, $C$ is stable and this completes the proof.

The previous result can be generalized to the case where $M$ is in a positive cone. Let

$$
P=\left\{M\left|M=\sum_{j=1}^{m} \gamma_{j} M_{j}\right| \gamma_{j} \geq 0, M \neq 0\right\}
$$

be a cone of matrices, which we refer to as a positive cone, then $P$ is said to be stable if each nonzero matrix in $P$ is stable. The following theorem follows.

THEOREM 2.3. If there exists a matrix measure $\mu$ such that $\mu\left(M_{j}\right)<0$ for $1 \leq j \leq m$, then $P$ is stable.

Proof. The matrix measure enjoys the following properties that can be found in [3]:

(1) $\mu(A+B) \leq \mu(A)+\mu(B)$ for any two matrices $A$ and $B$,

(2) $\mu(\alpha A)=\alpha \mu(A)$ for any $A$ and any $\alpha \geq 0$,

from which the proof of the theorem follows directly.

Although the conditions of Theorems 2.2 and 2.3 are only sufficient, for a certain class of vertices they become also necessary. Let $*$ denote the complex conjugate transpose operation, and let $S$ be a set of matrices. We say that $S$ is *-closed if $M^{*} \in S$ for any $M \in S$. This leads to the following theorem.

THEOREM 2.4. Let $V$ be the set of vertex matrices for a convex hull of matrices $C$, and let $V$ be $*$-closed. Then, $C$ is stable if and only if there exists a matrix measure $\mu$ such that $\mu(M)<0$ for any $M \in V$.

Proof. The sufficiency is guaranteed by Theorem 2.2. To prove necessity, we choose the vector 2-norm. For any $M \in V$, we have $M^{*} \in V$ since $V$ is $*$ closed. Since $C$ is stable, $\left(M+M^{*}\right) / 2$ is stable, that is, $\max _{j} \lambda_{j}\left(M+M^{*}\right) / 2<0$. From Lemma 2.1(c), we conclude that $\mu_{2}(M)<0$ and the proof is complete.

Of special interest is the following list of corollaries.

COROLLARY 2.5. If the vertex matrices are normal, then $C$ is stable if and only if $V$ is stable.

Proof. To prove sufficiency, let $M \in V$. Since $M$ is normal, then by [6, Theorem 2.5.4] $M$ can be diagonalized using a unitary transformation, that is, there exists a unitary matrix $U$ such that $M=U D U^{-1}$. Using the 2-norm, which is invariant under a unitary transformation, the induced matrix measure is also invariant; therefore,

$$
\mu_{2}(M)=\mu_{2}\left(U D U^{-1}\right)=\mu_{2}(D)=\max _{j} \operatorname{Re} \lambda_{j}(D)=\max _{j} \operatorname{Re} \lambda_{j}(M) .
$$


Hence, if $M$ is stable, then $\mu_{2}(M)<0$ for any $M \in V$, and from Theorem 2.2 we conclude that $C$ is stable.

Since necessity is trivial, the proof is then complete.

The main results of [12] can now be given in the next corollary, the proof of which follows directly from Theorem 2.4.

COROLLARY 2.6. If the vertex matrices are symmetric, then $C$ is stable if and only if $V$ is stable.

The next corollary represents one of the main results of [8], and can now be directly concluded from Theorem 2.2 by choosing the vector 2-norm.

Corollary 2.7. If for any $M \in V,\left(M+M^{T}\right) / 2$ is stable, then $C$ is stable.

A more general case than Corollary 2.7 is the following result.

COROLLARY 2.8. If there exists a positive matrix $H$ such that $H M+M^{T} H$ is stable for any $M \in V$, then $C$ is stable.

Proof. If $H$ is positive definite, let $H=K^{2}$, where $K$ is also positive definite; then, if we use the norm $\|x\|=\|K x\|_{2}$, the induced matrix measure $\mu_{K}$ is given by

$$
\begin{aligned}
\mu_{K}(M) & =\mu_{2}\left(K M K^{-1}\right)=\max _{j} \operatorname{Re} \lambda_{j}\left(\frac{K M K^{-1}+K^{-T} M^{T} K^{T}}{2}\right) \\
& =\max _{j} \operatorname{Re} \lambda_{j}\left(K^{-1} \frac{H M+M^{T} H}{2} K^{-1}\right) \leq \frac{\max _{j} \lambda_{j}\left(H M+M^{T} H\right)}{2 \alpha(H, M)},
\end{aligned}
$$

where $\alpha(H, M)=\max _{j} \lambda_{j}(H)$ if $H M+M^{T} H$ is negative semidefinite and $\alpha(H, M)=\min _{j} \lambda_{j}(H)$ if $H M+M^{T} H$ is positive semidefinite. If $H M+M^{T} H$ is stable for any $M \in V$, then it is negative definite; hence $\mu_{K}(M)<0$ for any $M \in V$. From Theorem 2.2, it follows that $C$ is stable.

Now, the main results of [1] follow directly from Theorem 2.2 as shown below.

COROLLARY 2.9. Let $C_{0}=\sum_{j=1}^{m} M_{j} / m$. Suppose that there exists a nonsingular $N$ such that $N C_{0} N^{-1}$ is diagonal. Then if there exists a matrix measure $\mu$ such that $\mu\left(N M_{j} N^{-1}\right)<0(1 \leq j \leq m), C$ is stable.

Proof. For any norm $|\cdot|$ with $N$ nonsingular, choose a new norm $\rho(x)=$ $|N x|$. The new induced matrix measure is $\mu_{\rho}(M)=\mu\left(N M N^{-1}\right)$ by Theorem 2.2, and the proof is complete.

The above results can be applied to the stability of interval matrices. Let $L=\left(l_{i j}\right), U=\left(u_{i j}\right)$, and $M=\left(m_{i j}\right)$ be real matrices, and let $L \leq M \leq U$ hold elementwise. 
Define

$$
\begin{aligned}
& K=\{M \mid L \leq M \leq U\}=\left\{\left(m_{i j}\right) \mid l_{i j} \leq m_{i j} \leq u_{i j}\right\}, \\
& V=\left\{M \mid m_{i j}=l_{i j} \text { or } m_{i j}=u_{i j}\right\}
\end{aligned}
$$

that is, $V$ is the set of vertex matrices of $K$. Then, the following result follows from Theorem 2.2.

COROLLARY 2.10. If there exists a matrix measure $\mu$ such that $\mu(M)<0$ for any $M \in V$, then $K$ is stable.

The criteria developed in this paper may seem restricted because the matrix measure is only an upper bound of the real parts of the eigenvalues of a matrix. The next theorem will establish a relationship between the real parts of the eigenvalues and the matrix measure, showing that if the matrix measure is properly chosen, then it will be possible to obtain stability criteria which are equivalent to testing the real parts of the eigenvalues for negativity.

THEOREM 2.11. Let $\mathcal{N}$ be the set of all vector norms on $\mathbb{C}^{n} ;$ for any $\rho \in \mathcal{N}$, the corresponding matrix measure is denoted by $\mu_{\rho}$; then for any matrix in $\mathbb{C}^{n \times n}$, we have

$$
\max _{1 \leq j \leq n} \operatorname{Re} \lambda_{j}(M)=\inf _{\rho \in \mathcal{N}} \mu_{\rho}(M)
$$

Proof. Let $J$ be the Jordan form of $M$, then from Jordan's theorem, there exists a nonsingular matrix $N$ such that $J=N M N^{-1}=D+U$, where $D$ is diagonal and $U$ is a matrix whose diagonal elements are zeros and off-diagonal elements are the same as $J$.

Let $\Lambda=\operatorname{diag}\left\{1, \sigma^{-1}, \sigma^{-2}, \ldots, \sigma^{-(n-1)}\right\}$ for any positive real $\sigma$, then

$$
\Lambda J \Lambda^{-1}=D+\sigma U
$$

Define a new norm of $\mathbb{C}^{n}$ by $|x|=|\Lambda N x|_{2}$. Then the corresponding matrix norm of $I+\gamma M$, where $I$ denotes the identity matrix and $\gamma$ is any positive number, is

$$
\|I+\gamma M\|=\max _{|x|=1}|(I+\gamma M) x|=\max _{|\Lambda N x|_{2}=1}|\Lambda N(I+\gamma M) x|_{2} .
$$

Note that if we let $z=\Lambda N x$, then we have

$$
\Lambda N(I+\gamma M) x=\Lambda N(I+\gamma M) N^{-1} \Lambda^{-1} z=I+\gamma(D+\sigma U) z
$$


so the corresponding matrix measure of $M$ is

$$
\begin{aligned}
\mu(M) & =\lim _{\gamma \rightarrow 0} \frac{\|I+\gamma M\|-1}{\gamma}=\lim _{\gamma \rightarrow 0} \frac{\max _{|z|_{2}=1}|z+\gamma(D+\sigma U) z|_{2}-1}{\gamma} \\
& \leq \lim _{\gamma \rightarrow 0} \frac{\max _{|z|_{2}=1}\left(|(I+\gamma D) z|_{2}+\sigma \gamma|U z|_{2}\right)-1}{\gamma}=\mu_{2}(D)+\sigma|U|_{2} \\
& \leq \max _{j} \operatorname{Re} \lambda_{j}(M)+\sigma .
\end{aligned}
$$

Therefore, for any positive real $\sigma$, there exists a norm $\rho$ such that

$$
\mu_{\rho}(M)<\max _{1 \leq j \leq n} \operatorname{Re} \lambda_{j}(M)+\sigma
$$

and the proof is thus complete.

The above theorem suggests that in order to obtain the tightest stability bounds, the norm in Theorem 2.2 must be chosen properly. One possible choice is the class given by the norms $|x|_{N}=|N x|_{2}$ for any nonsingular matrix $N$. The induced matrix measure is $\mu_{N}(M)=\mu_{2}\left(N M N^{-1}\right)$. This relates to the representation problem for the matrix $M$. For the stability of interval polynomials, it is interesting to note that the stability of the system depends on finding a suitable state space realization so that the results of Theorem 2.2 can be applied. Now, we state an obvious corollary of Theorem 2.11.

COROLLARY 2.12. The matrix $M$ is stable if and only if there exists a matrix measure $\mu$ such that $\mu(M)<0$.

Of special interest is the stability of time-invariant linear systems with parameter variations for unmodeled dynamics. Using the concept of the matrix measure, we can obtain the following result.

THEOREM 2.13. Suppose that the dynamical system is described by

$$
\dot{x}(t)=(M+\Delta M) x(t),
$$

where $\Delta M$ represents the unmodeled dynamics, then the system is stable if the unmodeled dynamics satisfies the conditions

$$
\mu(\Delta M)<-\mu(M)
$$

where $\mu$ is a certain matrix measure.

The proof follows directly from the property $\mu(A+B) \leq \mu(A)+\mu(B)$.

The next corollary shows that our results can be used to test stability of a convex hull of triangular vertex matrices. 
COROLLARY 2.14. If the vertex matrices can be simultaneously transformed by a similarity transformation to upper (or lower) triangular form, then a necessary and sufficient condition for $C$ to be stable is that the vertex matrices are stable.

Proof. Without loss of generality, we assume that all vertex matrices $M_{1}$, $M_{2}, \ldots, M_{r}$ are upper triangular. Let $M_{k}=\left(m_{i j}^{k}\right)(k=1,2, \ldots, r)$ and $D=$ $\operatorname{diag}\left\{d_{1}, d_{2}, \ldots, d_{n}\right\}$. Let $\mu$ denote the matrix measure induced by the vector norm $\left\|D^{-1} x\right\|_{\infty}$. Since

$$
D^{-1} M_{k} D=\left(\begin{array}{cccc}
m_{11}^{k} & \left(d_{2} / d_{1}\right) m_{12}^{k} & \cdots & \left(d_{n} / d_{1}\right) m_{1 n}^{k} \\
& m_{22}^{k} & \cdots & \left(d_{n} / d_{2}\right) m_{2 n}^{k} \\
& & \ddots & \vdots \\
& & & m_{n n}^{k}
\end{array}\right), \quad k=1,2, \ldots, r,
$$

we obtain from Lemma 2.1(c) and (d)

$$
\mu\left(M_{k}\right)=\mu_{\infty}\left(D^{-1} M_{k} D\right)=\max _{j}\left[\operatorname{Re}\left(m_{i i}^{k}+\sum_{j>i}\left(\frac{d_{j}}{d_{i}}\right)\left|m_{i j}^{k}\right|\right)\right] .
$$

Let $d_{j}=\sigma^{j}(j=0,1, \ldots, n-1)$, where $\sigma$ is a sufficiently small number. Define $S=\max _{1 \leq k \leq r} \max _{i, j}\left|m_{i j}^{k}\right|$. Then we have

$$
\mu\left(M_{k}\right) \leq \max _{j}\left[\operatorname{Re}\left(m_{i i}^{k}+S \sum_{j>i} \frac{d_{j}}{d_{i}}\right)\right] \leq \max _{i}\left[m_{i i}^{k}\right]+n S \sigma .
$$

Therefore, if $M_{k}$ is stable, then we can choose $\sigma>0$ sufficiently small so that $\mu\left(M_{k}\right)<0$ for any $k=1,2, \ldots, r$. It follows from Theorem 2.2 that $C$ is stable. The necessary part of the theorem is trivial.

COROLLARY 2.15. If the vertex matrices commute, then $C$ is stable if and only if the vertex matrices are stable.

Proof. By [6, Theorem 2.3.3], if a family of matrices commute, then they can be simultaneously transformed to upper triangular form. Then, Corollary 2.14 leads to the desired result.

We end up with the following corollary, whose proof is straightforward.

COROLLARY 2.16. If the vertex matrices can be simultaneously transformed to Jordan form, then $C$ is stable if and only if the vertex matrices are stable. 


\section{Examples}

EXAMPLE 3.1. Let

$$
M_{1}=\left(\begin{array}{cc}
0 & 1 \\
-2 & -3
\end{array}\right), \quad M_{2}=\left(\begin{array}{cc}
-12 & -3 \\
4 & 5
\end{array}\right), \quad N=\left(\begin{array}{cc}
2 & 1 \\
-1 & -1
\end{array}\right) .
$$

Then,

$$
N M_{1} N^{-1}=\left(\begin{array}{cc}
-1 & 0 \\
0 & -2
\end{array}\right), \quad N M_{2} N^{-1}=\left(\begin{array}{cc}
-9 & 2 \\
0 & -8
\end{array}\right)
$$

Define the vector norm $|x|=\left|N_{x}\right|_{2}$. Then the induced matrix measure is $\mu(M)=\mu_{2}\left(N M N^{-1}\right)$. Thus $\mu\left(M_{1}\right)=-1<0$ and $\mu\left(M_{2}\right)=-7.382<0$, and from Theorem 2.2, we conclude that $C$ is stable.

REMARK 3.2. Since $\mu_{2}\left(M_{1}\right)=0.081>0$, we cannot use Jiang's results [8]. Note also that for $D=\operatorname{diag}\left\{d_{1}, d_{2}\right\}, \mu_{D}(M)=\mu_{\infty}\left(D^{-1} M D\right)$, which is the matrix measure induced by the vector norm $\left\|D^{-1}\right\|_{\infty}$. Then for any positive numbers $d_{1}$ and $d_{2}$,

$$
\mu_{D}\left(M_{2}\right)=\mu_{\infty}\left(D^{-1} M_{2} D\right)=4\left(d_{1} / d_{2}\right)+5>0 .
$$

Hence, Heinen's results [5] fail the stability test.

EXAMPLE 3.3. Consider the interval dynamical system

$$
\dot{x}(t)=M x(t),
$$

where

$$
M \in M_{1}=\left(\begin{array}{cc}
{[-5,-3]} & {[1,2]} \\
{[4,5]} & {[-6,-4]}
\end{array}\right) .
$$

From the observation that

$$
\mu_{2}\left(\left(\begin{array}{cc}
-3 & 2 \\
7 & -4
\end{array}\right)\right)=\frac{1}{2}(-7+\sqrt{82})>0,
$$

it follows that the criterion which uses the 2-norm measure developed in [8] cannot be used to study the stability of $M_{I}$.

Choose $D=\operatorname{diag}\{1,0.5\}$. Then

$$
D M_{I} D^{-1}=\left(\begin{array}{cc}
{[-5,-3]} & {[2,4]} \\
{[2,2.5]} & {[-6,-4]}
\end{array}\right) .
$$

Let

$$
L=\left(\begin{array}{cc}
-5 & 2 \\
2 & -6
\end{array}\right), \quad U=\left(\begin{array}{cc}
-3 & 4 \\
2.5 & -4
\end{array}\right), \quad V=\left\{M \mid m_{i j}=l_{i j} u_{i j}\right\} .
$$


For all $M \in V$, the characteristic polynomial of $M^{T}+M$ is

$$
\lambda^{2}-2\left(m_{11}+m_{22}\right) \lambda+4 m_{11} m_{22}-\left(m_{12}+m_{21}\right)^{2} .
$$

Since $m_{11}<0$ and $m_{22}<0$, it follows that

$$
\mu_{D}(M)<0 \Longleftrightarrow M+M^{T} \text { stable } \Longleftrightarrow 4 m_{11} m_{22}-\left(m_{12}+m_{21}\right)^{2}>0 .
$$

For all $M \in V$,

$$
\begin{aligned}
& 4 m_{11} m_{22}-\left(m_{12}+m_{21}\right)^{2} \\
& \geq 4(-3)(-4)-(4+2.5)>0 \Longleftrightarrow \mu_{D}(M)<0 \Longleftrightarrow M_{I} \text { is stable. }
\end{aligned}
$$

Therefore, our criterion is applicable where that of [8] fails.

EXAMPLE 3.4. Consider

$$
u=\left\{\Delta M\left|\Delta M=\sum_{j=1}^{r} \alpha_{j} M_{j},\right| \alpha_{j} \mid \leq \beta_{j}, j=1,2, \ldots, r\right\},
$$

where the matrices $M_{i}$, which represent the uncertain structure, are given skew matrices, that is, $M_{j}^{T}=-M_{j}(j=1,2, \ldots, r)$. The problem is to find conditions such that $M+\Delta M$ is asymptotically stable for any $\Delta M \in \mathcal{U}$ (in this case, we say that $M+\mathcal{U}$ is stable), where $M$ satisfies $M+M^{T}$ is negative definite. The above formulation can be viewed as the representation of a dissipative system with energy-conserving perturbation. If we choose the 2-norm, the induced matrix measure is $\mu_{2}$, which satisfies $\mu_{2}(M+\Delta M)=(1 / 2) \lambda_{\max }\left(M+M^{T}\right)<0$; so under the above assumption, we conclude that $M+\mathcal{U}$ is stable with a convergence rate at least $-\mu_{2}(M)$.

4. Conclusion. In this paper, we have obtained a very general sufficient condition for the stability of a convex hull of matrices and a positive cone of matrices. For a certain class of vertex matrices, the sufficient condition becomes also necessary. Another sufficient condition for the stability of an interval dynamical system is given, and the relationship between the real parts of the eigenvalues of a matrix and its matrix measure is derived. Using the matrix measure, we have also found a result for the stability of time-invariant linear systems with parameter variations representing the unmodeled dynamics.

\section{REFERENCES}

[1] M. B. Argoun, On sufficient conditions for the stability of interval matrices, Internat. J. Control 44 (1986), no. 5, 1245-1250.

[2] K. J. Arrow and M. McManus, A note on dynamic stability, Econometrica 26 (1958), 448-454.

[3] C. A. Desoer and M. Vidyasagar, Feedback Systems: Input-Output Properties, Electrical Science, Academic Press, New York, 1975. 
[4] F. R. Gantmacher, The Theory of Matrices. Vol. 2, Chelsea Publishing, New York, 1959.

[5] J. A. Heinen, Sufficient conditions for stability of interval matrices, Internat. J. Control 39 (1984), no. 6, 1323-1328.

[6] R. A. Horn and C. R. Johnson, Matrix Analysis, Cambridge University Press, Cambridge, 1985.

[7] J. L. Howland, The method of quadratic forms in dynamic stability problems, Third Canadian Conference in Applied Mechanics (Calgary), 1971.

[8] C. L. Jiang, Sufficient condition for the asymptotic stability of interval matrices, Internat. J. Control 46 (1987), no. 5, 1803-1810.

[9] - Sufficient and necessary condition for the asymptotic stability of discrete linear interval systems, Internat. J. Control 47 (1988), no. 5, 1563-1565.

[10] V. L. Kharitonov, The asymptotic stability of the equilibrium state of a family of systems of linear differential equations, Differentsial'nye Uravneniya 14 (1978), no. 11, 2086-2088.

[11] J. Mawhin, Some remarks on the $\Omega$-stability for families of polynomials, Arch. Math. (Brno) 33 (1997), no. 1-2, 139-145.

[12] Z. C. Shi and W. B. Gao, A necessary and sufficient condition for the positivedefiniteness of interval symmetric matrices, Internat. J. Control 43 (1986), no. 1, 325-328.

[13] Z. Zahreddine, An extension of the Routh array for the asymptotic stability of a system of differential equations with complex coefficients, Appl. Anal. 49 (1993), no. 1-2, 61-72.

[14] _ On the $\Gamma$-stability of systems of differential equations in the Routh-Hurwitz and the Schur-Cohn cases, Bull. Belg. Math. Soc. Simon Stevin 3 (1996), no. 3, 363-368.

Ziad Zahreddine: Department of Basic Sciences, College of Arts and Sciences, University of Sharjah, Sharjah P.O. 27272, United Arab Emirates

E-mail address: ziadzahr@sharjah.ac.ae 


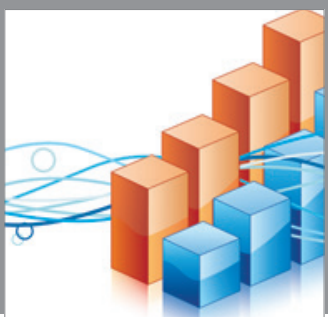

Advances in

Operations Research

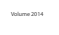

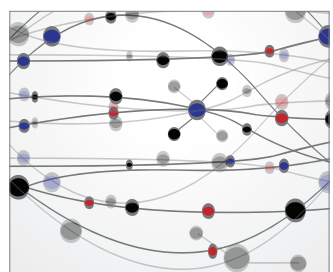

\section{The Scientific} World Journal
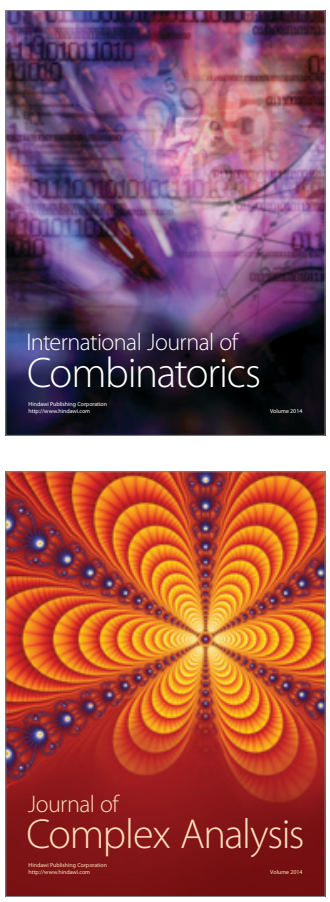

International Journal of

Mathematics and

Mathematical

Sciences
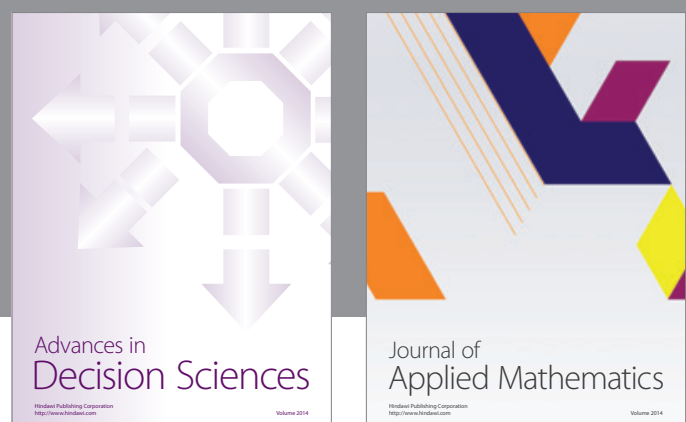

Journal of

Applied Mathematics
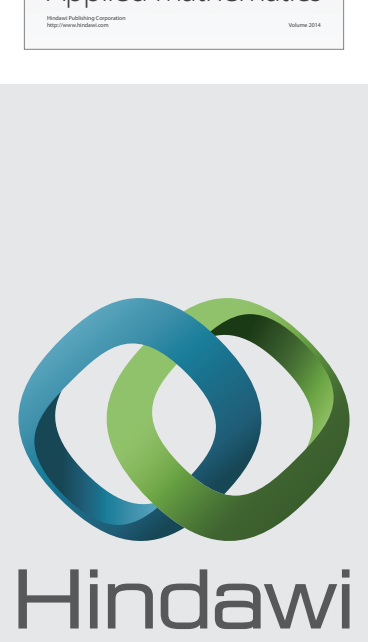

Submit your manuscripts at http://www.hindawi.com
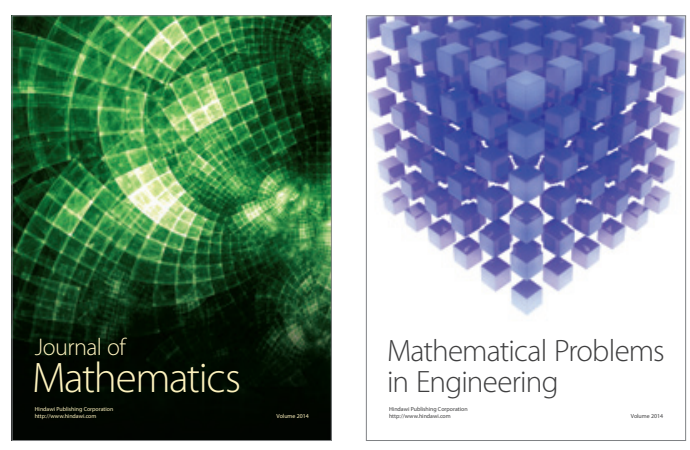

Mathematical Problems in Engineering
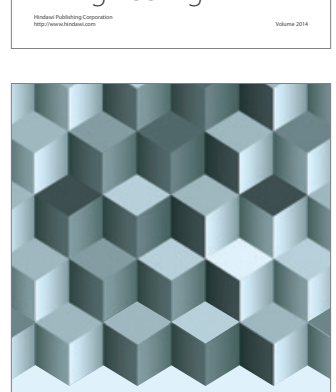

Journal of

Function Spaces
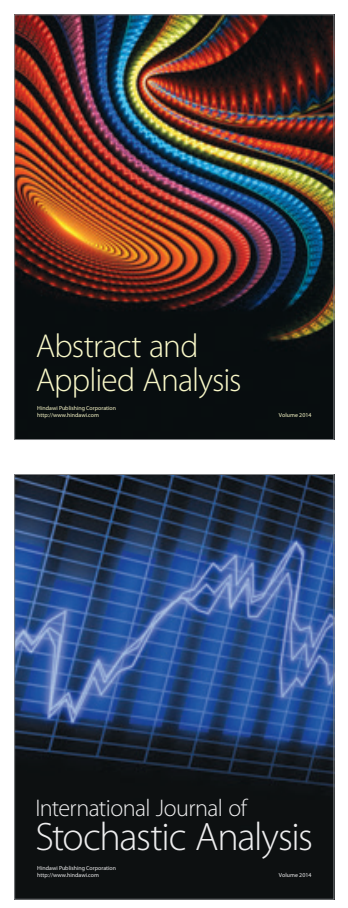

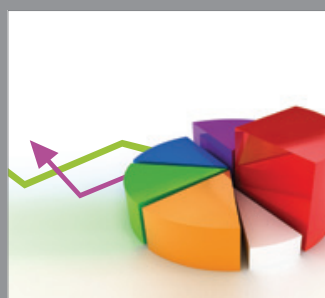

ournal of

Probability and Statistics

Promensencen
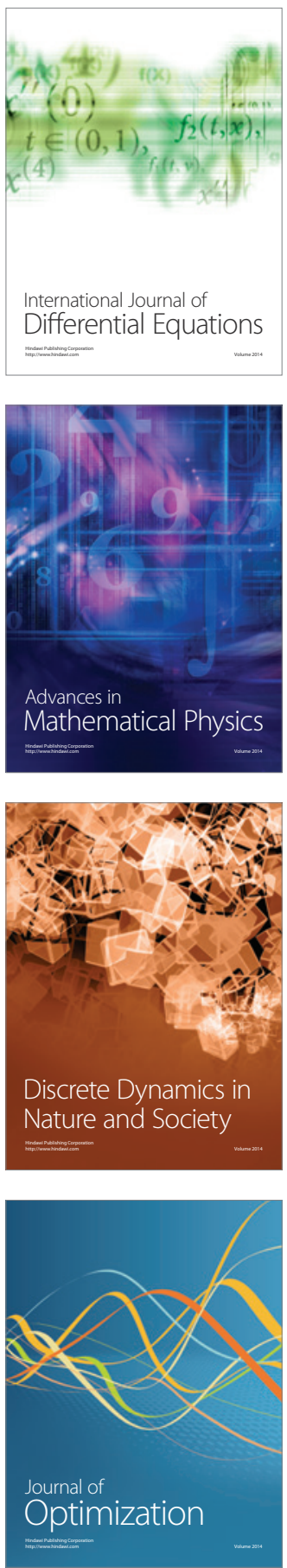